\title{
Refinements of some integral inequalities for unified integral operators
}

\author{
Chahn Yong Jung ${ }^{1}$, Ghulam Farid², Maja Andrić ${ }^{3}$, Josip Pečarić ${ }^{4}$ and Yu-Ming Chu ${ }^{5,6^{*}}$
}

"Correspondence:

chuyuming2005@126.com

${ }^{5}$ Department of Mathematics,

Huzhou University, Huzhou 313000,

P.R. China

${ }^{6}$ Hunan Provincial Key Laboratory of

Mathematical Modeling and

Analysis in Engineering, Changsha

University of Science \& Technology,

Changsha 410114, P.R. China

Full list of author information is

available at the end of the article

\begin{abstract}
In this paper we are presenting the refinements of integral inequalities established for convex functions. Consequently, we get refinements of several fractional integral inequalities for different kinds of fractional integral operators.
\end{abstract}

MSC: 26D10;31A10;26A33

Keywords: Integral operators; Fractional integrals; Convex functions; Bounds

\section{Introduction}

Integral operators are very useful in the theory of differential equations and boundary value problems. They are applied to formulate and solve mathematical models of real world problems; nowadays fractional integral operators are frequently studied to extend and generalize classical subjects. Fractional integral operators have converted the classical notions into modern concepts. In the recent past, fractional integral operators were utilized extensively to study the classical inequalities, see $[2,3,6,8,9,13,14,16-18,20,22]$ and the references therein.

The aim of this paper is to give several integral inequalities for strongly convex functions, resulting in refinements of the integral inequalities presented in [16], also [8, 9, 12]. For this purpose, we will need the following integral operators:

Definition 1 ([15]) Let $\tau_{1}:[a, b] \rightarrow \mathbb{R}$ be an integrable function. Also let $\tau_{2}$ be an increasing and positive function on $(a, b]$, having a continuous derivative $\tau_{2}^{\prime}$ on $(a, b)$. The leftand right-sided fractional integrals of a function $\tau_{1}$ with respect to another function $\tau_{2}$ on $[a, b]$ of order $\mu$ where $\Re(\mu)>0$ are defined by:

$$
{ }_{\tau_{2}}^{\mu} I_{a^{+}} \tau_{1}(x)=\frac{1}{\Gamma(\mu)} \int_{a}^{x}\left(\tau_{2}(x)-\tau_{2}(t)\right)^{\mu-1} \tau_{2}^{\prime}(t) \tau_{1}(t) d t, \quad x>a,
$$

and

$$
{ }_{\tau_{2}}^{\mu} I_{b^{-}} \tau_{1}(x)=\frac{1}{\Gamma(\mu)} \int_{x}^{b}\left(\tau_{2}(t)-\tau_{2}(x)\right)^{\mu-1} \tau_{2}^{\prime}(t) \tau_{1}(t) d t, \quad x<b,
$$

where $\Gamma(\cdot)$ is the gamma function.

(c) The Author(s) 2021. This article is licensed under a Creative Commons Attribution 4.0 International License, which permits use, sharing, adaptation, distribution and reproduction in any medium or format, as long as you give appropriate credit to the original author(s) and the source, provide a link to the Creative Commons licence, and indicate if changes were made. The images or other third party material in this article are included in the article's Creative Commons licence, unless indicated otherwise in a credit line to the material. If material is not included in the article's Creative Commons licence and your intended use is not permitted by statutory regulation or exceeds the permitted use, you will need to obtain permission directly from the copyright holder. To view a copy of this licence, visit http://creativecommons.org/licenses/by/4.0/. 
A $k$-analogue of the above definition is defined as follows:

Definition 2 ([1]) Let $\tau_{1}:[a, b] \rightarrow \mathbb{R}$ be an integrable function. Also let $\tau_{2}$ be an increasing and positive function on $(a, b]$, having a continuous derivative $\tau_{2}^{\prime}$ on $(a, b)$. The left- and right-sided fractional integrals of a function $\tau_{1}$ with respect to another function $\tau_{2}$ on $[a, b]$ of order $\mu ; \Re(\mu), k>0$ are defined by:

$$
\begin{aligned}
& { }_{\tau_{2}}^{\mu} I_{a^{+}}^{k} \tau_{1}(x)=\frac{1}{k \Gamma_{k}(\mu)} \int_{a}^{x}\left(\tau_{2}(x)-\tau_{2}(t)\right)^{\frac{\mu}{k}-1} \tau_{2}^{\prime}(t) \tau_{1}(t) d t, \quad x>a, \\
& { }_{\tau_{2}}^{\mu} I_{b^{-}}^{k} \tau_{1}(x)=\frac{1}{k \Gamma_{k}(\mu)} \int_{x}^{b}\left(\tau_{2}(t)-\tau_{2}(x)\right)^{\frac{\mu}{k}-1} \tau_{2}^{\prime}(t) \tau_{1}(t) d t, \quad x<b,
\end{aligned}
$$

where $\Gamma_{k}(\cdot)$ is defined by [5]

$$
\Gamma_{k}(x)=\int_{0}^{\infty} t^{x-1} e^{-\frac{t^{k}}{k}} d t, \quad \Re(x)>0 .
$$

The integral operators (1.3) and (1.4) produce several fractional integral operators, see [16, Remark 1]. A well-known Mittag-Leffler function is defined by [19]

$$
E_{\alpha}(z)=\sum_{n=0}^{\infty} \frac{z^{n}}{\Gamma(\alpha n+1)}
$$

where $\alpha, z \in \mathbb{C}$ and $\Re(\alpha)>0$.

This function has been extended and generalized in several different ways. In the following, we give a definition of an extended Mittag-Lefffler function with its corresponding fractional integral operator.

Definition 3 ([2]) Let $\omega, \mu, \alpha, l, \gamma, c \in \mathbb{C}, \Re(\mu), \Re(\alpha), \Re(l)>0, \Re(c)>\Re(\gamma)>0$ with $p \geq 0$, $\delta>0$ and $0<k \leq \delta+\Re(\mu)$. Let $\tau_{1} \in L_{1}[a, b]$ and $x \in[a, b]$. Then the generalized fractional integral operators $\epsilon_{\mu, \alpha, l, \omega, a^{+}}^{\gamma, \delta, k} \tau_{1}$ and $\epsilon_{\mu, \alpha, l, \omega, b^{-}}^{\gamma, \delta} \tau_{1}$ are defined by:

$$
\begin{aligned}
& \left(\epsilon_{\mu, \alpha, l, \omega, a^{+}}^{\gamma, \delta, k, c} \tau_{1}\right)(x ; p)=\int_{a}^{x}(x-t)^{\alpha-1} E_{\mu, \alpha, l}^{\gamma, \delta, k}\left(\omega(x-t)^{\mu} ; p\right) \tau_{1}(t) d t \\
& \left(\epsilon_{\mu, \alpha, l, \omega, b^{-}}^{\gamma, \delta, k, c} \tau_{1}\right)(x ; p)=\int_{x}^{b}(t-x)^{\alpha-1} E_{\mu, \alpha, l}^{\gamma, \delta, k, c}\left(\omega(t-x)^{\mu} ; p\right) \tau_{1}(t) d t
\end{aligned}
$$

where

$$
E_{\mu, \alpha, l}^{\gamma, \delta, k, c}(t ; p)=\sum_{n=0}^{\infty} \frac{\beta_{p}(\gamma+n k, c-\gamma)}{\beta(\gamma, c-\gamma)} \frac{(c)_{n k}}{\Gamma(\mu n+\alpha)} \frac{t^{n}}{(l)_{n \delta}}
$$

is the extended Mittag-Leffler function.

Recently, a unified integral operator was defined, which unifies several fractional integrals in a compact formula as follows:

Definition 4 ([11]) Let $\tau_{1}, \tau_{2}:[a, b] \longrightarrow \mathbb{R}, 0<a<b$, be functions such that $\tau_{1}$ is positive and $\tau_{1} \in L_{1}[a, b]$ and $\tau_{2}$ is differentiable and strictly increasing. Also let $\frac{\phi}{x}$ be an increasing 
function on $[a, \infty)$ and $\alpha, l, \gamma, c \in \mathbb{C}, \Re(\alpha), \Re(l)>0, \Re(c)>\Re(\gamma)>0, p, \mu, \delta \geq 0$, and $0<k \leq$ $\delta+\mu$. Then for $x \in[a, b]$, the left and right integral operators are defined by

$$
\begin{aligned}
& \left({ }_{\tau_{2}} F_{\mu, \alpha, l, a^{+}}^{\phi, \gamma} \tau_{1}\right)(x, \omega ; p)=\int_{a}^{x} K_{x}^{y}\left(E_{\mu, \alpha, l}^{\gamma, \delta, k, c}, \tau_{2} ; \phi\right) \tau_{1}(y) d\left(\tau_{2}(y)\right), \\
& \left({ }_{\tau_{2}} F_{\mu, \alpha, l, l, b^{-}}^{\phi, \gamma, c} \tau_{1}\right)(x, \omega ; p)=\int_{x}^{b} K_{y}^{x}\left(E_{\mu, \alpha, l}^{\gamma, \delta, k, c}, \tau_{2} ; \phi\right) \tau_{1}(y) d\left(\tau_{2}(y)\right),
\end{aligned}
$$

where $K_{x}^{y}\left(E_{\mu, \alpha, l}^{\gamma, \delta, k, c}, \tau_{2} ; \phi\right)=\frac{\phi\left(\tau_{2}(x)-\tau_{2}(y)\right)}{\tau_{2}(x)-\tau_{2}(y)} E_{\mu, \alpha, l}^{\gamma, \delta, k, c}\left(\omega\left(\tau_{2}(x)-\tau_{2}(y)\right)^{\mu} ; p\right)$.

For suitable settings of functions $\phi, \tau_{2}$ and certain values of parameters included in Mittag-Leffler function (1.9), very interesting consequences are obtained which are described in [16, Remarks 6 \& 7].

The objective of this paper is to obtain some inequalities for unified integral operators via strongly convex functions.

Definition 5 A function $\tau_{1}: I \rightarrow \mathbb{R}$, where $I$ is an interval in $\mathbb{R}$, is said to be convex if

$$
\tau_{1}(t x+(1-t) y) \leq t \tau_{1}(x)+(1-t) \tau_{1}(y)
$$

holds for all $x, y \in I$ and $t \in[0,1]$.

The following well-known Hadamard inequality holds for convex functions:

Definition 6 ([7]) Let $\tau_{1}: I \rightarrow \mathbb{R}$ be a convex function on an interval $I \subset \mathbb{R}$ and $a, b \in I$ where $a<b$. Then

$$
\tau_{1}\left(\frac{a+b}{2}\right) \leq \frac{1}{b-a} \int_{a}^{b} \tau_{1}(t) d t \leq \frac{\tau_{1}(a)+\tau_{1}(b)}{2}
$$

holds.

Definition 7 ([21]) Let $I$ be a nonempty convex subset of the normed space $\mathbf{X}$. A real valued function $\tau_{1}$ is said to be strongly convex with modulus $G>0$ on $I$ if for each $a, b \in I$ and $t \in[0,1]$,

$$
\tau_{1}(t x+(1-t) y) \leq t \tau_{1}(x)+(1-t) \tau_{1}(y)-G t(1-t)\|b-a\|^{2} .
$$

In the following, we give some results which are directly linked with the main findings of this paper. The following bounds of unified integral operators for convex functions are established in [16]:

Theorem 1 Let $\tau_{1}:[a, b] \longrightarrow \mathbb{R}$ be a positive integrable convex function with $m \in(0,1]$. Let $\tau_{2}:[a, b] \longrightarrow \mathbb{R}$ be differentiable and strictly increasing function, also let $\frac{\phi}{x}$ be an increasing function on $[a, b]$. If $\alpha, l, \gamma, c \in \mathbb{C}, \Re(\alpha), \Re(l)>0, \Re(c)>\Re(\gamma)>0, p, \mu, \delta \geq 0$, and $0<k \leq$ 
$\delta+\mu$, then for $x \in(a, b)$ we have

$$
\begin{aligned}
\left({ }_{\tau_{2}} F_{\mu, \alpha, l, a^{+}}^{\phi, \gamma, k} \tau_{1}\right)(x, \omega ; p)+\left({ }_{\tau_{2}} F_{\mu, \alpha, l, b^{-}}^{\phi, \gamma, \delta, k} \tau_{1}\right)(x, \omega ; p) \\
\leq K_{x}^{a}\left(E_{\mu, \alpha, l}^{\gamma, \delta, k, c}, \tau_{2} ; \phi\right)\left(\left(\tau_{2}(x)-\tau_{2}(a)\right)\left(\tau_{1}(x)+\tau_{1}(a)\right)\right) \\
\quad+K_{b}^{x}\left(E_{\mu, \beta, l}^{\gamma, \delta, k, c}, \tau_{2} ; \phi\right)\left(\left(\tau_{2}(b)-\tau_{2}(x)\right)\left(\tau_{1}(b)+\tau_{1}(x)\right)\right) .
\end{aligned}
$$

The following Hadamard inequality for unified fractional integrals is proved in [16]:

Theorem 2 Under the assumptions of Theorem 1, in addition if $\tau_{1}(x)=\tau_{1}(a+b-x)$, then we have

$$
\begin{aligned}
& \tau_{1}\left(\frac{a+b}{2}\right)\left(\left({ }_{\tau_{2}} F_{\mu, \alpha, l, b^{-}}^{\phi, \gamma, \delta, k, c} 1\right)(a, \omega ; p)+\left({ }_{\tau_{2}} F_{\mu, \alpha, l, a^{+}}^{\phi, \gamma, \delta, k, c} 1\right)(b, \omega ; p)\right) \\
& \quad \leq\left({ }_{\tau_{2}} F_{\mu, \alpha, l, b^{-}}^{\phi, \gamma, \delta, k, c} \tau_{1}\right)(a, \omega ; p)+\left({ }_{\tau_{2}} F_{\mu, \alpha, l, a^{+}}^{\phi, \gamma, \delta, k, c} \tau_{1}\right)(b, \omega ; p) \\
& \left.\quad \leq 2 K_{b}^{a}\left(E_{\mu, \alpha, l}^{\gamma, \delta, k, c}, \tau_{2} ; \phi\right)\right)\left(\tau_{2}(b)-\tau_{2}(a)\right)\left(\tau_{1}(b)+\tau_{1}(a)\right) .
\end{aligned}
$$

The following modulus inequality is obtained for unified integrals in [16].

Theorem 3 Let $\tau_{1}:[a, b] \longrightarrow \mathbb{R}$ be a differentiable function. Let $\left|\tau_{1}^{\prime}\right|$ be convex with $m \in$ $(0,1]$ and $\tau_{2}:[a, b] \longrightarrow \mathbb{R}$ be differentiable and strictly increasing function, also let $\frac{\phi}{x}$ be an increasing function on $[a, b]$. If $\alpha, l, \gamma, c \in \mathbb{C}, \mathfrak{R}(\alpha), \mathfrak{R}(l)>0, \mathfrak{R}(c)>\mathfrak{R}(\gamma)>0, p, \mu, \delta \geq 0$, and $0<k \leq \delta+\mu$, then for $x \in(a, b)$ we have

$$
\begin{aligned}
\left|\left(\tau_{2} F_{\mu, \alpha, l, a^{+}}^{\phi, \gamma, \delta, k} \tau_{1} * \tau_{2}\right)(x, \omega ; p)+\left({ }_{\tau_{2}} F_{\mu, \alpha, l, b^{-}}^{\phi, \gamma, \delta, k} \tau_{1} * \tau_{2}\right)(x, \omega ; p)\right| \\
\leq K_{x}^{a}\left(E_{\mu, \alpha, l}^{\gamma, \delta, k, c}, \tau_{2} ; \phi\right)\left(\left(\tau_{2}(x)-\tau_{2}(a)\right)\left(\left|\tau_{1}^{\prime}(x)\right|+\left|\tau_{1}^{\prime}(a)\right|\right)\right) \\
\quad+K_{b}^{x}\left(E_{\mu, \alpha, l}^{\gamma, \delta, k, c}, \tau_{2} ; \phi\right)\left(\left(\tau_{2}(b)-\tau_{2}(x)\right)\left(\left|\tau_{1}^{\prime}(b)\right|+\left|\tau_{1}^{\prime}(x)\right|\right),\right.
\end{aligned}
$$

where

$$
\begin{aligned}
& \left({ }_{2} F_{\mu, \alpha, l, a^{+}}^{\phi, \gamma, \delta, k, c} \tau_{1} * \tau_{2}\right)(x, \omega ; p):=\int_{a}^{x} K_{x}^{t}\left(E_{\mu, \alpha, l}^{\gamma, \delta, k, c}, \tau_{2} ; \phi\right) \tau_{1}^{\prime}(t) d\left(\tau_{2}(t)\right), \\
& \left({ }_{\tau_{2}} F_{\mu, \alpha, l, b^{-}}^{\phi, \gamma, \delta, k, c} \tau_{1} * \tau_{2}\right)(x, \omega ; p):=\int_{x}^{b} K_{t}^{x}\left(E_{\mu, \alpha, l}^{\gamma, \delta, k, c}, \tau_{2} ; \phi\right) \tau_{1}^{\prime}(t) d\left(\tau_{2}(t)\right) .
\end{aligned}
$$

In the upcoming section, we establish the bounds of a unified integral operator using strongly convexity. An Hadamard inequality is obtained for these integral operators via strongly convex function. A modulus inequality is obtained for differentiable functions by utilizing strongly convexity of $\left|\tau_{1}^{\prime}\right|$ for unified integral operators. Furthermore, refinements of results given in $[8,16]$ are identified. In the whole paper we will use

$$
I\left(a, b, \tau_{2}\right)=: \frac{1}{b-a} \int_{a}^{b} \tau_{2}(t) d t .
$$

\section{Main results}

Bounds of unified integral operators (1.10) and (1.11) are studied in the following result: 
Theorem 4 Let $\tau_{1}:[a, b] \longrightarrow \mathbb{R}$ be a positive, integrable and strongly convex function with $m \in(0,1]$. Let $\tau_{2}:[a, b] \longrightarrow \mathbb{R}$ be differentiable and strictly increasing function, also let $\frac{\phi}{x}$ be an increasingfunction on $[a, b]$. If $\alpha, \beta, l, \gamma, c \in \mathbb{C}, \Re(\alpha), \Re(l)>0, \Re(c)>\Re(\gamma)>0, p, \mu, \delta \geq 0$, and $0<k \leq \delta+\mu$, then for $x \in(a, b)$ we have

$$
\begin{aligned}
\left(\tau_{2} F_{\mu, \alpha, l, a^{+}}^{\phi, \gamma} \tau_{1}\right)(x, \omega ; p)+\left({ }_{\tau_{2}} F_{\mu, \beta, l, b b^{-}}^{\phi, \gamma, \delta, k, c} \tau_{1}\right)(x, \omega ; p) \\
\leq K_{x}^{a}\left(E_{\mu, \alpha, l}^{\gamma, \delta, k, c}, \tau_{2} ; \phi\right)\left(\left(\tau_{2}(x)-\tau_{2}(a)\right)\left(\tau_{1}(x)+\tau_{1}(a)\right)\right. \\
\left.\quad-G(x-a)\left(2 I\left(a, x, I_{d} \tau_{2}\right)-(a+x) I\left(a, x, \tau_{2}\right)\right)\right) \\
\quad+K_{b}^{x}\left(E_{\mu, \beta, l}^{\gamma, \delta, k, c}, \tau_{2} ; \phi\right)\left(\left(\tau_{2}(b)-\tau_{2}(x)\right)\left(\tau_{1}(b)+\tau_{1}(x)\right)\right. \\
\left.\quad-G(b-x)\left(2 I\left(x, b, I_{d} \tau_{2}\right)-(x+b) I\left(x, b, \tau_{2}\right)\right)\right),
\end{aligned}
$$

where $I_{d}$ is the identity function.

Proof Let $t \in(a, x)$. Then the following inequality holds:

$$
K_{x}^{t}\left(E_{\mu, \alpha, l}^{\gamma, \delta, k, c}, \tau_{2} ; \phi\right) \tau_{2}^{\prime}(t) \leq K_{x}^{a}\left(E_{\mu, \alpha, l}^{\gamma, \delta, k, c}, \tau_{2} ; \phi\right) \tau_{2}^{\prime}(t), \quad x \in(a, b) .
$$

Since $\tau_{1}$ is a strongly convex function, for $\tau_{1}$ the following inequality holds true:

$$
\tau_{1}(t) \leq\left(\frac{x-t}{x-a}\right) \tau_{1}(a)+\left(\frac{t-a}{x-a}\right) \tau_{1}(x)-G(x-t)(t-a) .
$$

Multiplying (2.2) with (2.3) and integrating over $[a, x]$, one can obtain

$$
\begin{aligned}
& \int_{a}^{x} K_{x}^{t}\left(E_{\mu, \alpha, l}^{\gamma, \delta, c, c}, \tau_{2} ; \phi\right) \tau_{1}(t) d\left(\tau_{2}(t)\right) \\
& \quad \leq K_{x}^{a}\left(E_{\mu, \alpha, l}^{\gamma, \delta, k, c}, \tau_{2} ; \phi\right)\left(\tau_{1}(a) \int_{a}^{x}\left(\frac{x-t}{x-a}\right) d\left(\tau_{2}(t)\right)+\tau_{1}(x) \int_{a}^{x}\left(\frac{t-a}{x-a}\right) d\left(\tau_{2}(t)\right)\right. \\
& \left.\quad-G \int_{a}^{x}(x-t)(t-a) d\left(\tau_{2}(t)\right)\right) .
\end{aligned}
$$

By using (1.10) of Definition 4 and integrating by parts, the following inequality is obtained:

$$
\begin{aligned}
& \quad\left({ }_{\tau_{2}} F_{\mu, \alpha, l, a^{+}}^{\phi, \gamma, c} \tau_{1}\right)(x, \omega ; p) \\
& \quad \leq K_{x}^{a}\left(E_{\mu, \alpha, l}^{\gamma, \delta, k, c}, \tau_{2} ; \phi\right)\left(\left(\tau_{2}(x)-\tau_{2}(a)\right)\right. \\
& \left.\left.\quad \times\left(\tau_{1}(x)+\tau_{1}(a)\right)\right)-G(x-a)\left(2 I\left(a, x, I_{d} \tau_{2}\right)-(a+x) I\left(a, x, \tau_{2}\right)\right)\right) .
\end{aligned}
$$

On the other hand, the following inequality holds true:

$$
K_{x}^{t}\left(E_{\mu, \alpha, l}^{\gamma, \delta, k, c}, \tau_{2} ; \phi\right) \tau_{2}^{\prime}(t) \leq K_{b}^{x}\left(E_{\mu, \beta, l}^{\gamma, \delta, k, c}, \tau_{2} ; \phi\right) \tau_{2}^{\prime}(t), \quad x \in(a, b) .
$$

Using strongly convexity of $\tau_{1}$, we have

$$
\tau_{1}(t) \leq\left(\frac{t-x}{b-x}\right) \tau_{1}(b)+\left(\frac{b-t}{b-x}\right) \tau_{1}(x)-G(t-x)(b-t) .
$$


Adopting the same procedure as we did for (2.2) and (2.3), the following inequality from (2.5) and (2.6) can be obtained:

$$
\begin{aligned}
& \left(\tau_{2} F_{\mu, \beta, l, b^{-}}^{\phi, \gamma, \delta, c} \tau_{1}\right)(x, \omega ; p) \\
& \quad \leq K_{b}^{x}\left(E_{\mu, \beta, l}^{\gamma, \delta, k, c}, \tau_{2} ; \phi\right) \\
& \quad \times\left(\left(\tau_{2}(b)-\tau_{2}(x)\right)\left(\tau_{1}(b)+\tau_{1}(x)\right)-G(b-x)\left(2 I\left(x, b, I_{d} \tau_{2}\right)-(x+b) I\left(x, b, \tau_{2}\right)\right)\right) .
\end{aligned}
$$

By adding (2.4) and (2.7), (2.1) is obtained.

Remark 1 (i) If we take $G=0$ in (2.1), then (1.14) is obtained. In other words, (2.1) provides a refinement of (1.14).

(ii) If we take $\phi(t)=\frac{\Gamma(\alpha) t \frac{\alpha}{k}}{k \Gamma_{k}(\alpha)}, G=0, \tau_{2}(x)=x$, and $p=\omega=0$ in (1.14), then [9, Theorem 1] is obtained. For $G \neq 0$, we get its refinement.

(iii) If we take $\alpha=\beta$ in the result of (ii), then [9, Corollary 1$]$ is obtained. For $G \neq 0$, we get its refinement.

(iv) If we take $\phi(t)=\frac{\Gamma(\alpha) t) \frac{\alpha}{k}}{k \Gamma_{k}(\alpha)}, G=0$, and $p=\omega=0$ in (1.14), then [12, Theorem 6] is obtained. For $G \neq 0$, we get its refinement.

(v) If we take $\alpha=\beta$ in the result of (iv), then [12, Corollary 7] is obtained. For $G \neq 0$, we get its refinement.

(vi) If we take $\phi(t)=\Gamma(\alpha) t^{\alpha}, G=0$, and $\tau_{2}(t)=t$ in (1.14), then [4, Corollary 1] is obtained. For $G \neq 0$, we get its refinement.

Corollary 1 If we take $\phi(t)=\Gamma(\alpha) t^{\alpha}, p=\omega=0$ in (2.1), then the following inequality is obtained for fractional integral operators defined in [15]:

$$
\begin{aligned}
\Gamma(\alpha) & I_{a^{+}}^{\alpha, \tau_{2}} \tau_{1}(x)+\Gamma(\beta) I_{b^{-}}^{\beta, \tau_{2}} \tau_{1}(x) \\
\leq & \left(\tau_{2}(x)-\tau_{2}(a)\right)^{\alpha-1}\left(\left(\tau_{1}(x) \tau_{2}(x)-\tau_{1}(a) \tau_{2}(a)\right)-\left(\tau_{1}(x)-\tau_{1}(a)\right) I\left(a, x, \tau_{2}\right)\right) \\
& +\left(\tau_{2}(b)-\tau_{2}(x)\right)^{\beta-1}\left(\left(\tau_{1}(b) \tau_{2}(b)-\tau_{1}(x) \tau_{2}(x)\right)-\left(\tau_{1}(b)-\tau_{1}(x)\right) I(x, b, g)\right) \\
& -G\left(( \tau _ { 2 } ( b ) - \tau _ { 2 } ( x ) ) ^ { \beta - 1 } ( b - x ) \left(2 I\left(x, b, I_{d} \tau_{2}\right)-(x+b) I\left(x, b, \tau_{2}\right)\right.\right. \\
& +\left(\tau_{2}(x)-\tau_{2}(a)\right)^{\alpha-1}(x-a)\left(2 I\left(a, x, I_{d} \tau_{2}\right)-(x+a) I\left(a, x, \tau_{2}\right)\right) .
\end{aligned}
$$

Remark 2 (i) If we take $G=0$ and $\alpha=\beta$ in (2.8), then [13, Theorem 1] is obtained. For $G \neq 0$, we get its refinement.

(ii) If $\alpha=\beta$ in the result of (i), then [13, Corollary 1$]$ is obtained. For $G \neq 0$, we get its refinement.

(iii) If we take $\tau_{2}(x)=x$ and $G=0$ in (2.8), then [8, Theorem 1] is obtained. For $G \neq 0$, we get its refinement.

(iv) If we take $\alpha=\beta$ in the result of (iii), then [8, Corollary 1] is obtained. For $G \neq 0$, we get its refinement.

(v) If we take $\alpha=\beta=1$ and $x=a$ or $x=b$ in the result of (iv), then [8, Corollary 2] is obtained. For $G \neq 0$, we get its refinement.

(vi) If we take $\alpha=\beta=1$ and $x=\frac{a+b}{2}$ in the result of (iv), then [8, Corollary 3] is obtained. For $G \neq 0$, we get its refinement. 
To prove the the next result we need the following lemma.

Lemma 1 Let $\tau_{1}:[a, b] \rightarrow \mathbb{R}$ be a strongly convex function. If $\tau_{1}$ is symmetric about $\frac{a+b}{2}$, then the following inequality holds true:

$$
\tau_{1}\left(\frac{a+b}{2}\right) \leq \tau_{1}(x)-\frac{G}{4}(a+b-2 x)^{2}
$$

for all $x \in[a, b]$.

Proof Since $\tau_{1}$ is strongly convex, we have

$$
\begin{aligned}
\tau_{1}\left(\frac{a+b}{2}\right) & \leq \frac{1}{2}\left[\tau_{1}\left(\frac{x-a}{b-a} b+\frac{b-x}{b-a} a\right)+\tau_{1}\left(\frac{x-a}{b-a} a+\frac{b-x}{b-a} b\right)\right]-\frac{G}{4}(a+b-2 x)^{2} \\
& =\frac{1}{2}\left(\tau_{1}(x)+\tau_{1}(a+b-x)\right)-\frac{G}{4}(a+b-2 x)^{2} .
\end{aligned}
$$

As $\tau_{1}$ is symmetric about $\frac{a+b}{2}$, we have $\tau_{1}(x)=\tau_{1}(a+b-x)$ and (2.9) holds.

Remark 3 Lemma 1 is a refinement of [8, Lemma 1].

The upcoming result gives the Hadamard inequality.

Theorem 5 Under the assumptions of Theorem 4, in addition if $\tau_{1}(x)=\tau_{1}(a+b-x)$, then we have

$$
\begin{aligned}
& \tau_{1}\left(\frac{a+b}{2}\right)\left({ }_{\tau_{2}} F_{\mu, \alpha, l, b^{-}}^{\phi, \gamma, \delta, k, c}\right)(a, \omega ; p)+\frac{G}{4}\left({ }_{\tau_{2}} F_{\mu, \alpha, l, b^{-}}^{\phi, \gamma, \delta, c}(a+b-2 x)^{2}\right)(a, \omega ; p) \\
& +\tau_{1}\left(\frac{a+b}{2}\right)\left({ }_{\tau_{2}} F_{\mu, \beta, l, a^{+}}^{\phi, \gamma}\right)(b, \omega ; p)+\frac{G}{4}\left(\tau_{2} F_{\mu, \beta, l, l, a^{+}}^{\phi, \gamma, \delta, c}(a+b-2 x)^{2}\right)(b, \omega ; p) \\
& \leq\left(\tau_{2} F_{\mu, \alpha, l, b^{-}}^{\phi, \gamma, \delta, k, c} \tau_{1}\right)(a, \omega ; p)+\left({ }_{\tau_{2}} F_{\mu, \beta, l, a^{+}}^{\phi, \gamma, c, c} \tau_{1}\right)(b, \omega ; p) \\
& \leq\left(K_{b}^{a}\left(E_{\mu, \alpha, l}^{\gamma, \delta, k, c}, \tau_{2} ; \phi\right)+K_{b}^{a}\left(E_{\mu, \beta, l}^{\gamma, \delta, k, c}, \tau_{2} ; \phi\right)\right) \\
& \times\left(\left(\tau_{2}(b)-\tau_{2}(a)\right)\left(\tau_{1}(b)+\tau_{1}(a)\right)-(b-a) G\left(2 I\left(a, b, I_{d} \tau_{2}\right)-(a+b) I(a, b, g)\right)\right) .
\end{aligned}
$$

Proof The following inequality holds true:

$$
K_{x}^{a}\left(E_{\mu, \alpha, l}^{\gamma, \delta, k, c}, \tau_{2} ; \phi\right) \tau_{2}^{\prime}(x) \leq K_{b}^{a}\left(E_{\mu, \alpha, l}^{\gamma, \delta, k, c}, \tau_{2} ; \phi\right) \tau_{2}^{\prime}(x), x \in(a, b) .
$$

Using strongly convexity of $\tau_{1}$ for $x \in(a, b)$, we have

$$
\tau_{1}(x) \leq\left(\frac{x-a}{b-a}\right) \tau_{1}(b)+\left(\frac{b-x}{b-a}\right) \tau_{1}(a)-G(x-a)(b-x) .
$$


Multiplying (2.11) and (2.12) and integrating the resulting inequality over $[a, b]$, one can obtain

$$
\begin{aligned}
\int_{a}^{b} & K_{x}^{a}\left(E_{\mu, \alpha, l}^{\gamma, \delta, k, c}, \tau_{2} ; \phi\right) \tau_{1}(x) d\left(\tau_{2}(x)\right) \\
\quad \leq & K_{b}^{a}\left(E_{\mu, \alpha, l}^{\gamma, \delta, k, c}, \tau_{2} ; \phi\right)\left(\tau_{1}(a) \int_{a}^{b}\left(\frac{b-x}{b-a}\right) d\left(\tau_{2}(x)\right)\right. \\
& \left.\quad+\tau_{1}(b) \int_{a}^{b}\left(\frac{x-a}{b-a}\right) d\left(\tau_{2}(x)\right)-G \int_{a}^{b}(b-x)(x-a) d\left(\tau_{2}(x)\right)\right) .
\end{aligned}
$$

By using Definition 4 and integrating by parts, the following inequality is obtained:

$$
\begin{aligned}
& \left(\tau_{2} F_{\mu, \alpha, l, b^{-}}^{\phi, \gamma, \delta} \tau_{1}\right)(a, \omega ; p) \\
& \leq K_{b}^{a}\left(E_{\mu, \alpha, l}^{\gamma, \delta, k, c}, \tau_{2} ; \phi\right) \\
& \quad \times\left(\left(\tau_{2}(b)-\tau_{2}(a)\right)\left(\tau_{1}(b)+\tau_{1}(a)\right)-G(b-a)\left(2 I\left(a, b, I_{d} \tau_{2}\right)-(a+b) I(a, b, g)\right)\right) .
\end{aligned}
$$

On the other hand, the following inequality holds true:

$$
K_{b}^{x}\left(E_{\mu, \beta, l}^{\gamma, \delta, k, c}, \tau_{2} ; \phi\right) \tau_{2}^{\prime}(x) \leq K_{b}^{a}\left(E_{\mu, \beta, l}^{\gamma, \delta, k, c}, \tau_{2} ; \phi\right) \tau_{2}^{\prime}(x), x \in(a, b) .
$$

Adopting the same pattern of simplification as we did for (2.11) and (2.12), the following inequality can be observed from (2.12) and (2.14):

$$
\begin{aligned}
& \left(\tau_{2} F_{\mu, \beta, l, a^{+}}^{\phi, \gamma} \tau_{1}\right)(b, \omega ; p) \\
& \leq K_{b}^{a}\left(E_{\mu, \beta, l}^{\gamma, \delta, k, c}, \tau_{2} ; \phi\right) \\
& \quad \times\left(\left(\tau_{2}(b)-\tau_{2}(a)\right)\left(\tau_{1}(b)+\tau_{1}(a)\right)-G(b-a)\left(2 I\left(a, b, I_{d} \tau_{2}\right)-(a+b) I(a, b, g)\right)\right) .
\end{aligned}
$$

By adding (2.13) and (2.15), the following inequality can be obtained:

$$
\begin{aligned}
& \left(\tau_{2} F_{\mu, \alpha, l, b-}^{\phi, \gamma, \delta, c} \tau_{1}\right)(a, \omega ; p)+\left({ }_{\tau_{2}} F_{\mu, \beta, l, a^{+}}^{\phi, \gamma, \delta, k, c} \tau_{1}\right)(b, \omega ; p) \\
& \leq\left(K_{b}^{a}\left(E_{\mu, \alpha, l}^{\gamma, \delta, k}, \tau_{2} ; \phi\right)+K_{b}^{a}\left(E_{\mu, \beta, l}^{\gamma, \delta, k, c}, \tau_{2} ; \phi\right)\right) \\
& \quad \times\left(\left(\tau_{2}(b)-\tau_{2}(a)\right)\left(\tau_{1}(b)+\tau_{1}(a)\right)-G(b-a)\left(2 I\left(a, b, I_{d} \tau_{2}\right)-(a+b) I(a, b, g)\right)\right) .
\end{aligned}
$$

Multiplying both sides of (2.9) by $K_{b}^{x}\left(E_{\mu, \beta, l}^{\gamma, \delta, k, c}, \tau_{2} ; \phi\right) d\left(\tau_{2}(x)\right)$ and integrating over $[a, b]$, we have

$$
\begin{aligned}
& \tau_{1}\left(\frac{a+b}{2}\right) \int_{a}^{b} K_{b}^{x}\left(E_{\mu, \beta, l}^{\gamma, \delta, k, c}, \tau_{2} ; \phi\right) d\left(\tau_{2}(x)\right) \\
& \quad \leq \int_{a}^{b} K_{b}^{x}\left(E_{\mu, \beta, l}^{\gamma, \delta, k, c}, \tau_{2} ; \phi\right) \tau_{1}(x) d\left(\tau_{2}(x)\right) \\
& \quad-\frac{G}{4} \int_{a}^{b} K_{b}^{x}\left(E_{\mu, \beta, l}^{\gamma, \delta, k, c}, \tau_{2} ; \phi\right)(a+b-2 x)^{2} d\left(\tau_{2}(x)\right) .
\end{aligned}
$$


From Definition 4, the following inequality is obtained:

$$
\begin{aligned}
\tau_{1}( & \left.\frac{a+b}{2}\right)\left({ }_{\tau_{2}} F_{\mu, \beta, l, a^{+}}^{\phi, \gamma, k, k} 1\right)(b, \omega ; p) \\
\leq & \left({ }_{\tau_{2}} F_{\mu, \beta, l, a^{+}}^{\phi, \gamma, \delta, k, c} \tau_{1}\right)(b, \omega ; p) \\
& \quad-\frac{G}{4}\left({ }_{\tau_{2}} F_{\mu, \beta, l, a^{+}}^{\phi, \gamma, \delta, k, c}(a+b-2 x)^{2}\right)(b, \omega ; p) .
\end{aligned}
$$

Similarly, multiplying both sides of (2.9) by $K_{x}^{a}\left(E_{\mu, \alpha, l}^{\gamma, \delta, k, c}, \tau_{2} ; \phi\right) d\left(\tau_{2}(x)\right)$ and integrating over $[a, b]$, we have

$$
\begin{aligned}
\tau_{1}( & \left.\frac{a+b}{2}\right)\left({ }_{\tau_{2}} F_{\mu, \alpha, l, b^{-}}^{\phi, \gamma, k, k} 1\right)(a, \omega ; p) \\
\leq & \left({ }_{\tau_{2}} F_{\mu, \alpha, l, b^{-}}^{\phi, \gamma, \delta, k, c} \tau_{1}\right)(a, \omega ; p) \\
& \quad-\frac{G}{4}\left({ }_{\tau_{2}} F_{\mu, \alpha, l, b^{-}}^{\phi, \gamma, \delta, k, c}(a+b-2 x)^{2}\right)(a, \omega ; p) .
\end{aligned}
$$

By adding (2.17) and (2.18), following inequality is obtained:

$$
\begin{aligned}
& \tau_{1}\left(\frac{a+b}{2}\right)\left({ }_{\tau_{2}} F_{\mu, \alpha, l, b^{-}}^{\phi, \gamma, \delta, k, c} 1\right)(a, \omega ; p)+\frac{G}{4}\left({ }_{\tau_{2}} F_{\mu, \alpha, l, b^{-}}^{\phi, \gamma, \delta, k, c}(a+b-2 x)^{2}\right)(a, \omega ; p) \\
& \quad+\tau_{1}\left(\frac{a+b}{2}\right)\left({ }_{\tau_{2}} F_{\mu, \beta, l, a^{+}}^{\phi, \gamma, k, k} 1\right)(b, \omega ; p)+\frac{G}{4}\left({ }_{\tau_{2}} F_{\mu, \beta, l, a^{+}}^{\phi, \gamma, \delta, k}(a+b-2 x)^{2}\right)(b, \omega ; p) \\
& \quad \leq\left({ }_{\tau_{2}} F_{\mu, \alpha, l, b^{-}}^{\phi, \gamma, \delta, k, c} \tau_{1}\right)(a, \omega ; p)+\left({ }_{\tau_{2}} F_{\mu, \beta, l, a^{+}}^{\phi, \gamma, \delta, k, c} \tau_{1}\right)(b, \omega ; p) .
\end{aligned}
$$

Using (2.16) and (2.19), inequality (2.10) can be established.

Remark 4 (i) If we take $G=0$ and $\alpha=\beta$ in (2.10), then (1.15) is obtained, for $G \neq 0$, we get its refinement.

(ii) If we take $\phi(t)=\Gamma(\alpha) t^{\alpha+1}, p=\omega=0$ and $G=0$ in (2.10), then [13, Theorem 3] is obtained. For $G \neq 0$, we get its refinement.

(iii) If $\alpha=\beta$ in the result of (ii), then [13, Corollary 3] is obtained. For $G \neq 0$, we get its refinement.

(iv) If we take $\phi(t)=\Gamma(\alpha) t^{\frac{\alpha}{k}+1}, G=0, \tau_{2}(x)=x$ and $p=\omega=0$ in (2.10), then [9, Theorem 3] is obtained. For $G \neq 0$, we get its refinement.

(v) If we take $\alpha=\beta$ in the result of (iv), then [9, Corollary 6] is obtained. For $G \neq 0$, we get its refinement.

(vi) If we take $\phi(t)=\Gamma(\alpha) t^{\frac{\alpha}{k}}+1, G=0$ and $p=\omega=0$ in (2.10), then [12, Theorem 11] is obtained. For $G \neq 0$, we get its refinement.

(vii) If we take $\alpha=\beta$ in the result of (vi), then [12, Corollary 12] is obtained. For $G \neq 0$, we get its refinement.

(viii) If we take $\phi(t)=t^{\alpha+1}, \tau_{2}(t)=t$ and $G=0$ in (2.10), then [4, Corollary 3] is obtained. For $G \neq 0$, we get its refinement. 
Corollary 2 If we take $p=\omega=0$ in (2.10), then the following Hadamard inequality is obtained for fractional integral operators defined in [10]:

$$
\begin{aligned}
\tau_{1}\left(\frac{a+b}{2}\right)\left(\frac{1}{\Gamma(\alpha)} F_{b^{-}}^{\phi, \tau_{2}} 1\right)(a)+\frac{G}{4}\left(\frac{1}{\Gamma(\alpha)} F_{b^{-}}^{\phi, \tau_{2}}(a+b-2 x)^{2}\right)(a) \\
\quad+\tau_{1}\left(\frac{a+b}{2}\right)\left(\frac{1}{\Gamma(\beta)} F_{a^{+}}^{\phi, \tau_{2}} 1\right)(b)+\frac{G}{4}\left(\frac{1}{\Gamma(\beta)} F_{a^{+}}^{\phi, \tau_{2}}(a+b-2 x)^{2}\right)(b) \\
\leq\left(\frac{1}{\Gamma(\alpha)} F_{b^{-}}^{\phi, \tau_{2}} \tau_{1}\right)(a)+\left(\frac{1}{\Gamma(\beta)} F_{a^{+}}^{\phi, \tau_{2}} \tau_{1}\right)(b) \\
\leq 2 K_{\tau_{2}}(t, x ; \phi) \\
\quad \times\left(\left(\tau_{2}(b)-\tau_{2}(a)\right)\left(\tau_{1}(b)+\tau_{1}(a)\right)-G(b-x)\left(2 I\left(x, b, I_{d} \tau_{2}\right)-(x+b) I\left(x, b, \tau_{2}\right)\right)\right) .
\end{aligned}
$$

Remark 5 (i) If we take $\phi(t)=\Gamma(\alpha) t^{\alpha+1}, p=\omega=0, G=0$, and $\tau_{2}(t)=t$ in (2.20), [8, Theorem 3] is obtained. For $G \neq 0$, we get its refinement.

(ii) If we take $\alpha=\beta$ in the result of (i), then [8, Corollary 6$]$ is obtained. For $G \neq 0$, we get its refinement.

Theorem 6 Let $\tau_{1}:[a, b] \longrightarrow \mathbb{R}$ be a differentiable function. Let $\left|\tau_{1}^{\prime}\right|$ be strongly convex with $m \in(0,1]$ and $\tau_{2}:[a, b] \longrightarrow \mathbb{R}$ be differentiable and strictly increasing function, also let $\frac{\phi}{x}$ be an increasing function on $[a, b]$. If $\alpha, \beta, l, \gamma, c \in \mathbb{C}, \Re(\alpha), \Re(l)>0, \Re(c)>\Re(\gamma)>0$, $p, \mu, \delta \geq 0$, and $0<k \leq \delta+\mu$, then for $x \in(a, b)$ we have

$$
\begin{aligned}
& \left|\left(\tau_{2} F_{\mu, \alpha, l, a^{+}}^{\phi, \gamma, \delta, c, c} \tau_{1} * \tau_{2}\right)(x, \omega ; p)+\left(\tau_{2} F_{\mu, \beta, l, b^{-}}^{\phi, \gamma, \delta, k, c} \tau_{1} * \tau_{2}\right)(x, \omega ; p)\right| \\
& \leq \quad K_{x}^{a}\left(E_{\mu, \alpha, l}^{\gamma, \delta, k, c}, \tau_{2} ; \phi\right) \\
& \quad \times\left(\left(\tau_{2}(x)-\tau_{2}(a)\right)\left(\left|\tau_{1}^{\prime}(x)\right|+\left|\tau_{1}^{\prime}(a)\right|\right)-G(x-a)\left(2 I\left(a, x, I_{d} \tau_{2}\right)-(a+x) I\left(a, x, \tau_{2}\right)\right)\right) \\
& \quad+K_{b}^{x}\left(E_{\mu, \beta, l}^{\gamma, \delta, k, c}, \tau_{2} ; \phi\right)\left(\left(\tau_{2}(b)-\tau_{2}(x)\right)\left(\left|\tau_{1}^{\prime}(b)\right|+\left|\tau_{1}^{\prime}(x)\right|\right)\right. \\
& \left.\quad-G(b-x)\left(2 I\left(x, b, I_{d} \tau_{2}\right)-(x+b) I\left(x, b, \tau_{2}\right)\right)\right) .
\end{aligned}
$$

Proof Using strongly convexity of $\left|\tau_{1}^{\prime}\right|$, we have

$$
\left|\tau_{1}^{\prime}(t)\right| \leq\left(\frac{x-t}{x-a}\right)\left|\tau_{1}^{\prime}(a)\right|+\left(\frac{t-a}{x-a}\right)\left|\tau_{1}^{\prime}(x)\right|-G(x-t)(t-a) .
$$

Inequality (2.22) can be written as follows:

$$
\begin{aligned}
& -\left(\left(\frac{x-t}{x-a}\right)\left|\tau_{1}^{\prime}(a)\right|+\left(\frac{t-a}{x-a}\right)\left|\tau_{1}^{\prime}(x)\right|-G(x-t)(t-a)\right) \\
& \quad \leq \tau_{1}^{\prime}(t) \\
& \quad \leq\left(\left(\frac{x-t}{x-a}\right)\left|\tau_{1}^{\prime}(a)\right|+\left(\frac{t-a}{x-a}\right)\left|\tau_{1}^{\prime}(x)\right|-G(x-t)(t-a)\right) .
\end{aligned}
$$

Let us consider the second inequality of (2.23), namely

$$
\tau_{1}^{\prime}(t) \leq\left(\frac{x-t}{x-a}\right)\left|\tau_{1}^{\prime}(a)\right|+\left(\frac{t-a}{x-a}\right)\left|\tau_{1}^{\prime}(x)\right|-G(x-t)(t-a) .
$$


Multiplying (2.2) and (2.24) and integrating over $[a, x]$, we can obtain

$$
\begin{aligned}
\int_{a}^{x} & K_{x}^{t}\left(E_{\mu, \alpha, l}^{\gamma, \delta, k, c}, \tau_{2} ; \phi\right) \tau_{1}^{\prime}(t) d\left(\tau_{2}(t)\right) \\
\leq & K_{x}^{a}\left(E_{\mu, \alpha, l}^{\gamma, \delta, k, c}, \tau_{2} ; \phi\right)\left(\left|\tau_{1}^{\prime}(a)\right| \int_{a}^{x}\left(\frac{x-t}{x-a}\right) d\left(\tau_{2}(t)\right)\right. \\
\quad & \left.+\left|\tau_{1}^{\prime}(x)\right| \int_{a}^{x}\left(\frac{t-a}{x-a}\right) d\left(\tau_{2}(t)\right)-G \int_{a}^{x}(x-t)(t-a) d\left(\tau_{2}(t)\right)\right) .
\end{aligned}
$$

By using (1.10) of Definition 4 and integrating by parts, the following inequality is obtained:

$$
\begin{aligned}
& \left({ }_{\tau_{2}} F_{\mu, \alpha, l, a^{+}}^{\phi, \gamma, \delta, k,} \tau_{1} * \tau_{2}\right)(x, \omega ; p) \\
& \quad \leq K_{x}^{a}\left(E_{\mu, \alpha, l}^{\gamma, \delta, k, c}, \tau_{2} ; \phi\right)\left(\left(\tau_{2}(x)-\tau_{2}(a)\right)\right. \\
& \left.\quad \times\left(\left|\tau_{1}^{\prime}(x)\right|+\left|\tau_{1}^{\prime}(a)\right|\right)-G(x-a)\left(2 I\left(a, x, I_{d} \tau_{2}\right)-(a+x) I\left(a, x, \tau_{2}\right)\right)\right) .
\end{aligned}
$$

If we consider the left-hand side of inequality (2.23) and adopt the same argument as for the right-hand side inequality, then we get

$$
\begin{aligned}
&\left({ }_{\tau_{2}} F_{\mu, \alpha, l, a^{+}}^{\phi, \gamma, \delta, k, c}\left(\tau_{1} * \tau_{2}\right)\right)(x, \omega ; p) \\
& \geq-K_{x}^{a}\left(E_{\mu, \alpha, l}^{\gamma, \delta, k, c}, \tau_{2} ; \phi\right)\left(\left(\tau_{2}(x)-\tau_{2}(a)\right)\right. \\
& \quad\left.\times\left(\left|\tau_{1}^{\prime}(x)\right|+\left|\tau_{1}^{\prime}(a)\right|\right)-G(x-a)\left(2 I\left(a, x, I_{d} \tau_{2}\right)-(a+x) I\left(a, x, \tau_{2}\right)\right)\right) .
\end{aligned}
$$

From (2.25) and (2.26), the following inequality is obtained:

$$
\begin{aligned}
& \left|\left(\tau_{2} F_{\mu, \alpha, l, a^{+}}^{\phi, \gamma, \delta, c}\left(\tau_{1} * \tau_{2}\right)\right)(x, \omega ; p)\right| \\
& \quad \leq K_{x}^{a}\left(E_{\mu, \alpha, l}^{\gamma, \delta, k, c}, \tau_{2} ; \phi\right)\left(\left(\tau_{2}(x)-\tau_{2}(a)\right)\right. \\
& \left.\quad \times\left(\left|\tau_{1}^{\prime}(x)\right|+\left|\tau_{1}^{\prime}(a)\right|\right)-G(x-a)\left(2 I\left(a, x, I_{d} \tau_{2}\right)-(a+x) I\left(a, x, \tau_{2}\right)\right)\right) .
\end{aligned}
$$

Now using the strongly convexity of $\left|\tau_{1}^{\prime}\right|$, we have

$$
\left|\tau_{1}^{\prime}(t)\right| \leq\left(\frac{t-x}{b-x}\right)\left|\tau_{1}^{\prime}(b)\right|+\left(\frac{b-t}{b-x}\right)\left|\tau_{1}^{\prime}(x)\right|-G(t-x)(b-t) .
$$

With the same procedure as that used for (2.2) and (2.22), one can obtain the following inequality from (2.5) and (2.28):

$$
\begin{aligned}
& \left|\left(\tau_{2} F_{\mu, \beta, l, b^{-}}^{\phi, \gamma, \delta, k}\left(\tau_{1} * \tau_{2}\right)\right)(x, \omega ; p)\right| \\
& \quad \leq K_{b}^{x}\left(E_{\mu, \beta, l}^{\gamma, \delta, k, c}, \tau_{2} ; \phi\right)\left(\left(\tau_{2}(b)-\tau_{2}(x)\right)\right. \\
& \left.\quad \times\left(\left|\tau_{1}^{\prime}(b)\right|+\left|\tau_{1}^{\prime}(x)\right|\right)-G(b-x)\left(2 I\left(x, b, I_{d} \tau_{2}\right)-(x+b) I\left(x, b, \tau_{2}\right)\right)\right) .
\end{aligned}
$$

By adding (2.27) and (2.29), inequality (2.21) can be achieved. 
Remark 6 (i) If we take $G=0$ and $\alpha=\beta$ in (2.21), then (1.16) is obtained. For $G \neq 0$, we get its refinement.

(ii) If we take $\phi(t)=\Gamma(\alpha) t^{\frac{\alpha}{k}+1}, G=0, \tau_{2}(x)=x$ and $p=\omega=0$ in (2.21), then [9, Theorem 2] is obtained. For $G \neq 0$, we get its refinement.

(iii) If we take $\alpha=\beta$ in the result of (ii), then [9, Corollary 4] is obtained. For $G \neq 0$, we get its refinement.

(iv) If we take $\alpha=\beta=k=1$ and $x=\frac{a+b}{2}$, in the result of (iii), then [9, Corollary 5] is obtained. For $G \neq 0$, we get its refinement.

(v) If we take $\phi(t)=t^{\alpha+1}, \tau_{2}(x)=x, p=\omega=0$ and $G=0$ in (2.21), then [8, Theorem 2] is obtained. For $G \neq 0$, we get its refinement.

(vi) If we take $\alpha=\beta$ in the result of (v), then [8, Corollary 5] is obtained. For $G \neq 0$, we get its refinement.

(vii) If we take $\phi(t)=\Gamma(\alpha) t^{\frac{\alpha}{k}+1}, \tau_{2}(x)=x, p=\omega=0$ and $G=0$ in (2.21), then [12, Theorem 8 ] is obtained. For $G \neq 0$, we get its refinement.

(viii) If we take $\alpha=\beta$ in the result of (vii), then [12, Corollary 9] is obtained. For $G \neq 0$, we get its refinement.

(ix) If we take $\phi(t)=t^{\alpha}, \tau_{2}(x)=x$ and $G=0$ in (2.21), then [4, Corollary 2] is obtained. For $G \neq 0$, we get its refinement.

\section{Concluding remarks}

This research provides inequalities for unified integral operators for strongly convex functions, refined form of convex functions. These inequalities provide refinements of the results proved in already published works. The special cases also provide results for fractional integral operators and their refinements.

\section{Acknowledgements}

The research work of Josip Pečaric is supported by the Ministry of Education and Science of the Russian Federation (Agreement No. 02.a03.21.0008). The research work of Yu-Ming Chu is supported by Chongqing University of Arts and Sciences (Z2018SC09) and the National Natural Science Foundation of China (Grant Nos. 11971142, 11871202, 61673169, 11701176,11626101 , and 11601485).

Funding

There is no funding available for the publication of this paper.

Availability of data and materials

There is no additional data required for the finding of results of this paper.

Competing interests

The authors declare that they have no competing interests.

Authors' contributions

All authors have equal contribution in this article. All authors read and approved the final manuscript.

\section{Author details}

${ }^{1}$ Department of Business Administration, Gyeongsang National University, Jinju 52828, Korea. ${ }^{2}$ Department of Mathematics, COMSATS University Islamabad, Attock Campus, Pakistan. ${ }^{3}$ Faculty of Civil Engineering, Architecture and Geodesy, University of Split, Matice hrvatske 15, 21000 Split, Croatia. ${ }^{4}$ Rudn University, Moscow, Russia. ${ }^{5}$ Department of Mathematics, Huzhou University, Huzhou 313000, P.R. China. ${ }^{6}$ Hunan Provincial Key Laboratory of Mathematical Modeling and Analysis in Engineering, Changsha University of Science \& Technology, Changsha 410114, P.R. China.

\section{Publisher's Note}

Springer Nature remains neutral with regard to jurisdictional claims in published maps and institutional affiliations. 


\section{References}

1. Akkurt, A., Yildirim, M.E., Yildirim, H.: On some integral inequalities for $(k, h)$-Riemann-Liouville fractional integral. New Trends Math. Sci. 4(2), 138-146 (2016)

2. Andrić, M., Farid, G., Pečarić, J.: A further extension of Mittag-Leffler function. Fract. Calc. Appl. Anal. 21(5), 1377-1395 (2018)

3. Chen, H., Katugampola, U.N.: Hermite-Hadamard and Hermite-Hadamard-Fejér type inequalities for generalized fractional integrals. J. Math. Anal. Appl. 446, 1274-1291 (2017)

4. Chen, Z., Farid, G., Rehman, A.U., Latif, N.: Estimations of fractional integral operators for convex functions and related results. Adv. Differ. Equ. 2020, 163 (2020)

5. Diaz, A.R., Parigunan, E.: On hypergeometric functions and k-Pochhammar symbol. Divulg. Mat. 15(2), 179-192 (2007)

6. Dragomir, S.S.: Inequalities of Jensens type for generalized $k-g$-fractional integrals of functions for which the composite $\tau_{1} \circ g^{-1}$ is convex. RGMIA Res. Rep. Collect. 20, Art. 133, 24 pp. (2017)

7. Dragomir, S.S., Pearce, C.E.M.: Selected topics on Hermite-Hadamard inequalities and applications, Mathematics Preprint Archive, (1), 463817 (2003)

8. Farid, G.: Some Riemann-Liouville fractional integral for inequalities for convex functions. J. Anal. (2018). https://doi.org/10.1007/s41478-0079-4

9. Farid, G.: Estimation of Riemann-Liouville k-fractional integrals via convex functions. Acta Comment. Univ. Tartu Math. 23(1), 71-78 (2019)

10. Farid, G.: Existence of an integral operator and its consequences in fractional and conformable integrals. Open J. Math. Sci. 3(3), 210-216 (2019)

11. Farid, G.: A unified integral operator and its consequences. Open J. Math. Anal. 4(1), 1-7 (2020)

12. Farid, G.: Study of generalized Riemann-Liouville fractional integral via convex functions. Commun. Fac. Sci. Univ. Ank. Sér. A1 Math. Stat. 69(1), 37-48 (2020)

13. Farid, G., Nazeer, W., Saleem, M.S., Mehmood, S., Kang, S.M.: Bounds of Riemann-Liouville fractional integrals in general form via convex functions and their applications. Mathematics 6, 248 (2018)

14. Huang, C.J., Rahman, G., Nisar, K.S., Ghafar, A., Qi, F.: Some inequalities of the Hermite-Hadamard type for k-fractional conformable integrals. Aust. J. Math. Anal. Appl. 16(1), Article 7, 9 pages (2019)

15. Kilbas, A.A., Srivastava, H.M., Trujillo, J.J.: Theory and Applications of Fractional Differential Equations. North-Holland Mathematics Studies, vol. 204. Elsevier, New York (2006)

16. Kwun, Y.C., Farid, G., Ullah, S., Nazeer, W., Mahreen, K., Kang, S.M.: Inequalities for a unified integral operator and associated results in fractional calculus. IEEE Access 7, 126283-126292 (2019)

17. Mehmood, S., Farid, G., Khan, K.A., Yussouf, M.: New fractional Hadamard and Fejér-Hadamard inequalities associated with exponentially $(h, m)$-convex functions. Lett. Appl. Eng. Sci. 3(2), 9-18 (2020)

18. Mehmood, S., Farid, G., Khan, K.A., Yussouf, M.: New Hadamard and Fejér-Hadamard fractional inequalities for exponentially m-convex function. Lett. Appl. Eng. Sci. 3(1), 45-55 (2020)

19. Mittag-Leffler, G.: Sur la nouvelle fonction $E_{\alpha}(x)$. C. R. Acad. Sci. Paris 137, 554-558 (1903)

20. Nisar, K.S., Tassaddiq, S., Rehman, G., Khan, A.: Some inequalities via fractional conformable integral operators. J. Inequal. Appl. 2019, 217 (2019)

21. Polyak, B.T.: Existence theorems and convergence of minimizing sequences in extremum problems with restrictions. Sov. Math. Dokl. 7, 72-75 (1966)

22. Rahman, G., Khan, A., Abdeljwad, T., Nisar, K.S.: The Minkowski inequalities via generalized proportional fractional integral operators. Adv. Differ. Equ. 2019, 287 (2019)

\section{Submit your manuscript to a SpringerOpen ${ }^{\circ}$ journal and benefit from:}

- Convenient online submission

- Rigorous peer review

- Open access: articles freely available online

- High visibility within the field

- Retaining the copyright to your article

Submit your next manuscript at $\boldsymbol{s p r i n g e r o p e n . c o m ~}$ 